\title{
A DISCUSSION ON THE HÖLDER AND ROBUST FINITE-TIME PARTIAL STABILIZABILITY OF BROCKETT'S INTEGRATOR*
}

\author{
Chaker JAMMAZI ${ }^{1}$
}

\begin{abstract}
We consider chained systems that model various systems of mechanical or biological origin. It is known according to Brockett that this class of systems, which are controllable, is not stabilizable by continuous stationary feedback (i.e. independent of time). Various approaches have been proposed to remedy this problem, especially instationary or discontinuous feedbacks. Here, we look at another stabilization strategy (by continuous stationary or discontinuous feedbacks) to ensure the asymptotic stability even in finite time for some variables, while other variables do converge, and not necessarily toward equilibrium. Furthermore, we build feedbacks that permit to vanish the two first components of the Brockett integrator in finite time, while ensuring the convergence of the last one. The considering feedbacks are continuous and discontinuous and regular outside zero.
\end{abstract}

Mathematics Subject Classification. 93D15, 93C10, 93D09.

Received January 21, 2010. Revised November 22, 2010.

Published online April 13, 2011.

\section{INTRODUCTION}

The problem of feedback stabilization of nonlinear control systems has been intensively studied and a considerable attention has been paid from the control community during the last decades. For further background and motivation, the reader is referred to $[7,23,42,60]$, and references therein.

One of the most challenging topics in this area is the design of local or global stabilizing feedback laws for the "without drift systems". Such systems have less actuator than the degree of freedom, and are part of underactuated system class. The without drift systems model real life applications, for example, mobile robots with steering wheels (unicycle), the underwater vehicle, the jumping robots, etc.

Since the two last decades, it has been shown that the linear control theory tools are not sufficient and stabilization techniques need to be reconsidered. Indeed, Brockett in [15] proved that all nonlinear controllable and differentiable systems cannot be stabilized by continuous state feedback laws. This obstruction is now called Brockett's necessary condition for stabilizability. This condition is generalized by Ryan for only continuous control systems [56]. The author in [56] proved that the Brockett's condition is still necessary for stabilizability

Keywords and phrases. Brockett's integrator, discontinuous feedback law, finite-time partial stability, rational partial stability, robust control.

* The paper is dedicated to Professor Jean-Michel Coron for his 54 th birthday.

${ }^{1}$ Faculté des Sciences de Bizerte, Département de Mathématiques and Laboratoire d'Ingénierie Mathématique, École Polytechnique de Tunisie, Université de Carthage, Avenue de la République, BP 77, 1054 Amilcar, Tunisia.

Chaker.Jammazi@ept.rnu.tn 
by discontinuous feedback laws in Ryan's sense. A strong homology necessary condition for stabilizability by dynamic feedback laws was given by Coron in [19]. The latter condition gave a negative answer to the question raised by Hermes in [31]. This question is: the Brockett condition and small time local controllability at zero imply the stabilizability? The Coron's condition can justify the non-stabilization of some systems when the Brockett condition is fulfilled.

In order to overcome the Brockett's condition for many nonlinear controllable systems especially systems without drift, or systems with drift as some underactuated marine and aerospace systems such as the autonomous underwater vehicle, the ship, the rigid spacecraft, the airship, etc.; three approaches have been proposed:

(1) Time-varying periodic controllers $[9,20,22,24,25,46-48,53,57,58,61]$;

(2) Discontinuous feedback laws [5,6,17,27,64];

(3) Partial asymptotic or finite-time stabilization by differentiable or continuous static feedback laws [36-40].

In this paper, we will focus our attention on the third approach. It consists of the concept of the partial asymptotic stabilization (PAS), this concept means the asymptotic stabilization with respect to the maximum components of the system while the remaining components are convergent to some positions depending on the initial conditions. The PAS concept is interesting from a practical point of view, see for instance [36,37,40].

It was proved in [40] that this theory is a natural extension of the classical concept of stability in Lyapunov sense. Stability with respect to the part called also "partial stability" has been intensively studied since Lyapunov in 1898, see for example $[28,29,55,65,68]$.

Basic results in this field are proposed in $[28,29,55,65,66]$. Moreover, the applicability of these results for the differential equations with continuous right side have solved many problems attached to distribute-parameter systems.

It is worth noting that the proposed definition of PAS in $[36,40]$ is different from those given and used in $[28,29,65,68]$, where the authors focused on a part of the system and supposed that the rest is bounded. The definition used in this paper takes into consideration the complete stability of the system, the asymptotic stability with respect to part of the state while the rest converges to some position depending on the initial conditions, the latter property is interesting since it removes the possible oscillations of the system. This PAS is applied in many engineering fields like the partial attitude control of rigid spacecraft with two controls, the partial asymptotic stabilization of the ship [36], the partial orientation of the autonomous underwater vehicle [41] and the finite-time partial stability of chained systems [37].

In the context of stabilization problem, the finite-time stabilization is the strongest and has been the subject of numerous papers and books $[7,22,23,30,32,33]$. Roughly speaking, the finite-time stabilizability consist in designing a feedback controller which renders the closed loop system Lyapunov stable and all trajectories converge to the origin in finite-time.

Recently, finite-time stability and finite-time stabilization via continuous time-invariant feedback laws have been studied and finite-time controllers involving terms containing fractional powers were constructed for second order systems, see [12,14,30,35,49]. Furthermore, output feedback finite-time control was also studied [33,34].

In [37], we proved that is possible to obtain a partial stabilization in finite-time of all chained systems by non-Lipschitz continuous state feedback laws. The controllers can stabilize in finite-time the $(n-1)$ first components of the chain of integrators while the last component remains constant for a large enough time. In [40], we derived a sufficient Lyapunov condition that guarantees the partial finite-time stability of all nonlinear dynamical systems. The obtained result is applied for several control systems as the ship, Sontag's example [60] and Coron's planar example [19]. For the ship system, we proposed continuous and homogeneous feedbacks that render four components finite-time stable. This leads to the exponential stability of one variable and the convergence of the remain state, these feedbacks appear stronger than one established in [36]. The same result is obtained, for the ship, if we proceed by discontinuous feedbacks and quasi-homogeneous properties.

In this paper, we propose three strategies for the partial asymptotic stabilization of the Brockett's integrator. The first one is the partial asymptotic stabilization by Hölder feedback laws, this approach makes the Brockett's integrator two-asymptotically stable (see Defs. 1.1 and 2.5) while the other state converges, this partial stabilization is termed "rational partial stability". Since, the partial rational stability is not robust under 


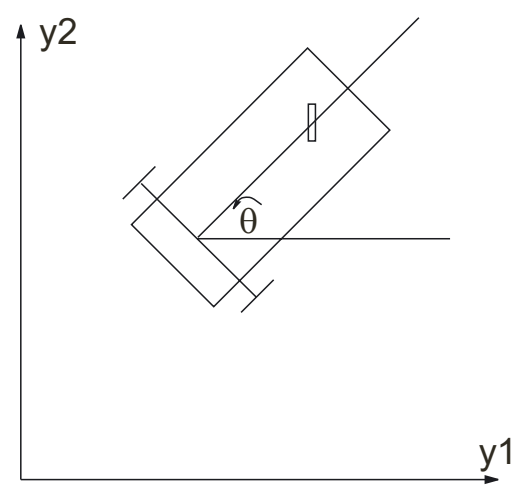

Figure 1. Unicycle system.

small disturbance the proposed second strategy is the partial stability in finite-time. By using the feedbacks cited in $[11,12,14]$ for the stabilization of the double integrator by homogeneous feedbacks, we show that the Brockett's integrator is two-stable in finite-time by continuous and homogeneous feedback laws (Def. 1.2) while the other state remains constant for a large time. The third approach is the partial stabilization in finite-time by discontinuous feedback laws [51] (Def. 5.1); we show as the second strategy, that, based on the discontinuous Orlov's feedback law for the double integrator [51] the unicycle is two-partially stable in finite-time by means of family of discontinuous feedback laws. Numerical simulations are given to illustrate our approaches and justify that this special stabilization is in many practical situations sufficient.

The paper is organized as follows: in Section 1 the benchmark Brockett's integrator and the partial stabilizability in finite-time are introduced.

In Section 2, we give a Hölder stabilizing feedback laws of the unicycle; which make the study system twopartially globally asymptotically stable. These feedback laws are obtained by using backstepping techniques and by asymptotic estimation of all trajectories of the system.

In Section 3, we give a similar version of Bhat and Bernstein [14] theorem concerning the finite-time stability of homogeneous systems in the case of partial stability in finite-time by homogeneous feedback laws. As an application of the obtained result, we give a family of explicit homogeneous feedback law that renders the unicycle two-partially stable in finite-time. Furthermore, we show that the size of the "convergence" or the limit of the uncontrollable part is bounded by an arbitrary positive real number which can be small enough.

Section 4 studies the robust stabilization of a perturbed unicycle system; the perturbed terms are attached to the velocity of the midpoint. We conjecture that for every small enough $\varepsilon$, the established feedback laws can stabilize locally in finite-time the perturbed midpoint, while the orientation $\theta$ remains constant for a large time.

In Section 5, we study the same problem by discontinuous state feedback laws. Our construction uses the extended invariance principle and the quasi-homogeneous properties for discontinuous right-hand side [51], and we guess that the stabilizing feedbacks are also robust with respect to small disturbance.

\section{BenCHMARK EXAMPLE}

A classical benchmark example of systems without drift is the unicycle system [57,59] (see Fig. 1) described in the equations:

$$
\dot{y}_{1}=u_{1} \cos \theta, \dot{y}_{2}=u_{1} \sin \theta, \dot{\theta}=u_{2},
$$

the state is given by the orientation $\theta$, together with the coordinates $\left(y_{1}, y_{2}\right)$ of the midpoint between the back wheels. $u=\left(u_{1}, u_{2}\right) \in \mathbb{R}^{2}$ is the control. We consider the control $u_{1}$ as a "drive command" and $u_{2}$ as a steering control. The stabilization problem of the unicycle is well studied in a number of publications. The first one is established by Samson [57] where the conception of time-varying feedback laws stabilizing the unicycle are demonstrated. See also $[24,26]$. 
It is well known that the system (1.1) is globally controllable but cannot be stabilized by stationary feedback laws from the classical Brockett's condition. As cited in the famous book of Coron [23], to get around the problem of impossibility to stabilize many controllable systems by continuous feedback laws, two main strategies have been proposed:

- Asymptotic stabilization by discontinuous feedback laws-see e.g. the pioneer work by Sussmann [64] as well as $[17,18,27]$, and the references therein;

- Asymptotic stabilization by continuous time-varying feedback laws - see e.g. the pioneer works by Sontag and Sussmann [61] and by Samson [57] as well as [20,22-24,26,46] and the references therein.

The main goal of this article is to propose a new strategy based on the construction of continuous feedback laws that stabilize the first components asymptotically or in finite-time while the orientation $\theta$ of the unicycle converges.

In order to state our main result, we first introduce some definitions.

\subsection{Basic notations and definitions}

In this section, the concept of partial asymptotic stability (respectively, stabilizability, finite-time stability) with related definitions and notations will be reviewed. Hereafter $\mathbb{R}$ and $\mathbb{R}^{n}$ denote the real numbers set and $n \times 1$ real column vectors set respectively. The Euclidean vector norm is noted by $||,. \mathcal{L}^{1}\left(\mathbb{R}_{+}\right)$denotes the integrable space in Lebesgue sense on $\mathbb{R}_{+}$and ' is the symbol of transposition.

Consider the following system:

$$
\left\{\begin{array}{l}
\dot{x}_{1}=X_{1}\left(x_{1}, x_{2}\right) \\
\dot{x}_{2}=X_{2}\left(x_{1}, x_{2}\right),
\end{array}\right.
$$

where $X=\left(X_{1}, X_{2}\right) \in C^{\infty}\left(\mathbb{R}^{p} \times \mathbb{R}^{n-p}, \mathbb{R}^{n}\right), x_{1} \in \mathbb{R}^{p}, x_{2} \in \mathbb{R}^{n-p}$ and $p$ is positive integer such that $0<p \leq n$. We assume that

$$
\forall x_{2} \in \mathbb{R}^{n-p}, X_{1}\left(0, x_{2}\right)=0, X_{2}\left(0, x_{2}\right)=0
$$

Definition 1.1 ( $p$-partial asymptotic stability [36]). The system (1.2) is said to be $p$-partially asymptotically stable if the following properties are satisfied:

a) The origin of the system (1.2) is Lyapunov stable.

b) The system (1.2) is asymptotically stable with respect to $x_{1}$ and $x_{2}$ converges, i.e.

$$
\exists r>0:\left(\left|x_{1}(0)\right|+\left|x_{2}(0)\right| \leq r\right) \Rightarrow\left\{\begin{array}{l}
\lim _{t \rightarrow+\infty} x_{1}(t)=0, \\
\lim _{t \rightarrow+\infty} x_{2}(t)=a,
\end{array}\right.
$$

where $a$ is a constant vector depending on the initial conditions.

The control system $\dot{x}=X(x, u)$ is $p$-partially asymptotically stabilizable if there exists a continuous feedback $x \mapsto u(x)$ such that, for every $x_{2} \in \mathbb{R}^{n-p}, u\left(0, x_{2}\right)=0$, and such that $(0,0) \in \mathbb{R}^{p} \times \mathbb{R}^{n-p}$ is $p$-partially asymptotically stable for the closed loop system $\dot{x}=X(x, u(x))$.

Definition 1.2 (p-finite-time partial stability [37]). Let $X: \mathbb{R}^{p} \times \mathbb{R}^{n-p} \cong \mathbb{R}^{n} \rightarrow \mathbb{R}^{n}, x=\left(x_{1}, x_{2}\right) \in \mathbb{R}^{p} \times \mathbb{R}^{n-p} \mapsto$ $X(x) \in \mathbb{R}^{n}$, be defined and continuous on a neighborhood of $(0,0) \in \mathbb{R}^{p} \times \mathbb{R}^{n-p}$. We assume that

$$
X\left(0, x_{2}\right)=0
$$


One says that $(0,0) \in \mathbb{R}^{p} \times \mathbb{R}^{n-p}$ is $p$-partially stable in finite time for $\dot{x}=X(x)$ if:

- $(0,0)$ is Lyapunov stable for $\dot{x}=X(x)$;

- there exists $r>0$ and, $T=T(x(0))>0$ called settling-time function, such that, if $\dot{x}=X(x)$ and $|x(0)|<r$, then $x_{1}(t)=0$ for every $t \geq T$ (and therefore $t \mapsto x_{2}(t)$ is constant for $t \geq T$ ).

The solution $x(t)$ starting from the initial condition $x(0)$ is defined and unique in forward time for $t \in[0, T)$.

The control system $\dot{x}=X(x, u)$ is $p$-partially stabilizable in finite time if there exists a continuous feedback $x \mapsto u(x)$ such that, for every $x_{2} \in \mathbb{R}^{n-p}, u\left(0, x_{2}\right)=0$, and such that $(0,0) \in \mathbb{R}^{p} \times \mathbb{R}^{n-p}$ is $p$-partially stable in finite-time for the closed loop system $\dot{x}=X(x, u(x))$.

\section{HÖLDER STABILIZING FEEDBACK LAWS OF THE UNICYCLE}

In this section, we give a Hölder stabilizing feedback laws that renders the unicycle system 2-partially asymptotically stabilizable. In some cases, the decay of the energy or the Lyapunov function is not exponential, but can be polynomial. Our aim here is to give a sufficient feedback law that yields the explicit decay rate.

Let the system described by equations (1.1). As usual, let us introduce the new coordinates as in [59]

$$
\begin{aligned}
& x_{1}=y_{1} \sin \theta-y_{2} \cos \theta, \\
& x_{2}=y_{1} \cos \theta+y_{2} \sin \theta, \\
& x_{3}=\theta, \\
& v_{1}=u_{2}, \quad v_{2}=u_{1}-x_{1} u_{2} .
\end{aligned}
$$

A simple calculation yields to well known Brockett's integrator

$$
\dot{x}_{1}=v_{1} x_{2}, \dot{x}_{2}=v_{2}, \dot{x}_{3}=v_{1} .
$$

Note that $\left(y_{1}, y_{2}\right)=0$ is equivalent to $\left(x_{1}, x_{2}\right)=0$. Our principal objective is to construct continuous feedback laws $v_{1}$ and $v_{2}$ such that

$$
v_{1}((0,0), \theta)=v_{2}((0,0), \theta)=0
$$

and

$$
\left\{\begin{array}{l}
\left(x_{1}, x_{2}\right) \rightarrow(0,0), \\
x_{3}=\theta \rightarrow \theta_{\infty} \in(-\infty, \infty) .
\end{array}\right.
$$

Our feedback construction is based on backstepping techniques [23]. The construction is done over two steps:

Step 1: We are interested to system (2.2) with respect to $\left(x_{1}, x_{2}\right)$ and we apply the backstepping techniques. We begin by the stabilization of the system

$$
\dot{x}_{1}=v_{1} v_{2} .
$$

This stabilization is obtained under the following feedback laws $v_{1}=x_{1}$ and $v_{2}=-x_{1}^{2 p}$ where $0<p<1 / 2$ is an odd rational number i.e. $p=\frac{p_{1}}{p_{2}}, p_{1}$ and $p_{2}$ are non-negative odd integers.

Step 2: Backstepping with respect to $x_{2}$.

Let $k \in \mathbb{N}^{*}$, and let the candidate Lyapunov function

$$
V=\frac{1}{2 k} x_{1}^{2 k}+\frac{1}{2}\left(x_{2}+x_{1}^{2 p}\right)^{2} .
$$


The time derivative of $V$ along the system (2.2) is given by

$$
\begin{aligned}
\dot{V} & =x_{1}^{2 k-1} x_{2} v_{1}+\left(x_{2}+x_{1}^{2 p}\right)\left(v_{2}+2 p x_{1}^{2 p-1} x_{2} v_{1}\right), \\
& =x_{1}^{2 k} x_{2}+\left(x_{2}+x_{1}^{2 p}\right)\left(v_{2}+2 p x_{1}^{2 p} x_{2}\right) .
\end{aligned}
$$

Let $y=x_{2}+x_{1}^{2 p}$. Then $x_{2}=y-x_{1}^{2 p}$.

A simple calculation yields

$$
\dot{V}=-x_{1}^{2(k+p)}+y\left(v_{2}+2 p x_{1}^{2 p} x_{2}+x_{1}^{2 k}\right) .
$$

It is then sufficient to choose

$$
v_{2}=-2 p x_{1}^{2 p} x_{2}-x_{1}^{2 k}-\alpha y^{1+\frac{2 p}{k}}, \quad \alpha>1 .
$$

Thus we get

$$
\dot{V}=-x_{1}^{2(k+p)}-\alpha y^{2+\frac{2 p}{k}},
$$

and the global asymptotic stabilization of $(2.2)$ with respect to $\left(x_{1}, x_{2}\right)$ follows from the first Lyapunov theorem.

\subsection{Asymptotic estimation of the solutions of (2.2)}

In order to get, under the feedback law $v_{1}$ in step 1 and $v_{2}$ in (2.23), the convergence of the sate $x_{3}$, the following section introduces the asymptotic estimation of all solutions of the system (2.2).

Proposition 2.1. There exists a constant $c>0$ such that

$$
\dot{V} \leq-c V^{1+\frac{p}{k}} .
$$

Proof. We have shown that the system (2.2) is globally asymptotically stabilizable with respect to $\left(x_{1}, x_{2}\right)$, and under the feedback law (2.7), we have

$$
-\dot{V}=x_{1}^{2(k+p)}+\alpha y^{2+\frac{2 p}{k}}
$$

then

The last inequality implies

$$
-\dot{V} \geq x_{1}^{2(k+p)} \text { and }-\dot{V} \geq \alpha y^{2+\frac{2 p}{k}} \geq y^{2+\frac{2 p}{k}}
$$

$$
(-\dot{V})^{\frac{k}{k+p}} \geq x_{1}^{2 k} \text { and }(-\dot{V})^{\frac{k}{k+p}} \geq y^{2} .
$$

From (2.9), we have

$$
2(-\dot{V})^{\frac{k}{k+p}} \geq x_{1}^{2 k}+y^{2} \geq V
$$

therefore we get

$$
\dot{V} \leq-(1 / 2)^{\frac{k+p}{k}} V^{\frac{k+p}{k}} .
$$

This inequality leads to the estimate $(2.8)$, where $c=(1 / 2)^{1+\frac{p}{k}}$.

Proposition 2.2. There exist two constants $a$ and $b$ depending on initial condition such that

$$
V \leq \frac{1}{(a t+b)^{\frac{k}{p}}} .
$$


Proof. The proof is obtained by integration of the differential inequality (2.8).

We have for $V \neq 0$,

$$
\frac{\mathrm{d}}{\mathrm{d} t} V^{-p / k} \geq \frac{c p}{k},
$$

then by integration the inequality (2.14) along $[0, t]$, we get

$$
V \leq \frac{1}{(a t+b)^{\frac{k}{p}}} \text {, where } a=\frac{c p}{k} \text { and } b=\frac{1}{V(x(0))^{p / k}} .
$$

The convergence of the state $x_{3}$ is based on the following classical lemma.

Lemma 2.3. Let $f$ be a differentiable function defined on $[0,+\infty)$ such that $\int_{0}^{+\infty}\left|f^{\prime}(s)\right| \mathrm{d} s<+\infty$ then $\lim _{t \rightarrow+\infty} f(t)$ exists.

Proof. Considering $x, y \in[0,+\infty)$ such that $x<y$, then we have

$$
|f(x)-f(y)|=\left|\int_{x}^{y} f^{\prime}(s) \mathrm{d} s\right| \leq \int_{x}^{y}\left|f^{\prime}(s)\right| \mathrm{d} s,
$$

by the assumption $\int_{0}^{+\infty}\left|f^{\prime}(s)\right| \mathrm{d} s<+\infty$, we get

$$
\lim _{x, y \rightarrow+\infty} \int_{x}^{y}\left|f^{\prime}(s)\right| \mathrm{d} s=0
$$

then the Cauchy condition for the limit is satisfied and therefore $\lim _{t \rightarrow+\infty} f(t)$ exists.

Proposition 2.4. If $0<2 p<1$, then $x_{1} \in \mathcal{L}^{1}\left(\mathbb{R}_{+}\right)$and therefore the state $x_{3}$ converges.

Proof. From (2.5), we have $x_{1}^{2 k} \leq 2 k V$, then by using Proposition 2.2, we get

$$
\left|x_{1}(t)\right| \leq \frac{c_{k}}{(a t+b)^{1 / 2 p}}, c_{k}=(2 k)^{1 / 2 k}
$$

It is clear that if $0<2 p<1$, then the state $x_{1}$ is Lebesgue integrable on $\mathbb{R}_{+}$. Indeed, we have $\int_{1}^{+\infty} \mathrm{d} t / t^{\alpha}$ converges if and only if $\alpha>1$. Since $\frac{1}{2 p}>1$, then from integral comparison theorem, we get $\int_{1}^{+\infty}\left|x_{1}(t)\right| \mathrm{d} t<$ $+\infty$, therefore

$$
\int_{0}^{+\infty}\left|x_{1}(t)\right| \mathrm{d} t=\int_{0}^{1}\left|x_{1}(t)\right| \mathrm{d} t+\int_{1}^{+\infty}\left|x_{1}(t)\right| \mathrm{d} t<+\infty .
$$

Since, the state $x_{3}(t)$ satisfies the equation $\dot{x}_{3}=v_{1}=x_{1}$ and $x_{1} \in \mathcal{L}^{1} \in\left(\mathbb{R}_{+}\right)$, then the convergence of $x_{3}$ follows from Lemma 2.3.

The Hölder stabilizing feedback laws obtained for the Brockett's integrator, are included in the so called also rational stability [8], can be generalized for the partial asymptotic stability as follows. 
Definition 2.5 (p-rational partial asymptotic stability). Let the dynamical system (1.2) with the assumption (1.3). The system (1.2) is said to be $p$-rational partially asymptotically stable if the following properties are satisfied:

a) the origin of the system (1.2) is Lyapunov stable;

b) there exist positive numbers $M, k, \eta$ and $r$ with $\eta \leq 1$ such that if

$$
\exists r>0:\left(\left|x_{1}(0)\right|+\left|x_{2}(0)\right| \leq r\right) \Rightarrow\left\{\begin{array}{l}
\left|x_{1}(t)\right| \leq \frac{M|x(0)|^{\eta}}{\left(1+|x(0)|^{k} t\right)^{k}}, \\
\lim _{t \rightarrow+\infty} x_{2}(t)=a(x(0)) .
\end{array}\right.
$$

The control system $\dot{x}=X(x, u)$ is $p$-rationally partially asymptotically stabilizable if there exists a continuous feedback $x \mapsto u(x)$ such that, for every $x_{2} \in \mathbb{R}^{n-p}, u\left(0, x_{2}\right)=0$, and such that $(0,0) \in \mathbb{R}^{p} \times \mathbb{R}^{n-p}$ is $p$-rationally partially asymptotically stable for the closed loop system $\dot{x}=X(x, u(x))$.

Clearly, Definition 2.5 of rational-partial asymptotic stability implies Definition 1.1 of the partial asymptotic stability.

The following theorem is inspired from the pioneer work of Hahn [8], and establish sufficient condition for the system 1.2 to be $p$-rational-partially asymptotically stable.

Theorem 2.6. Let the dynamical system (1.2) with the assumption (1.3), we assume that:

(1) there exists a $C^{1}$ function $V: \mathbb{R}^{p} \times \mathbb{R}^{n-p} \longrightarrow \mathbb{R}$ satisfying:

(i) there exist real numbers $c_{1}, c_{2}, c_{3}, r_{1}, r_{2}, r_{3} \in(0,+\infty)$ and $r_{3}>r_{2}$, such that for every $x=$ $\left(x_{1}, x_{2}\right) \in \mathbb{R}^{p} \times \mathbb{R}^{n-p}$ such that $|x|<\varepsilon$,

$$
c_{1}\left|x_{1}\right|^{r_{1}} \leq V\left(x_{1}, x_{2}\right) \leq c_{2}\left|x_{1}\right|^{r_{2}}
$$

(ii) for every $x=\left(x_{1}, x_{2}\right) \in \mathbb{R}^{p} \times \mathbb{R}^{n-p}$ such that $|x|<\varepsilon$,

$$
\dot{V}\left(x_{1}, x_{2}\right) \leq-c_{3}\left|x_{1}\right|^{r_{3}}, \forall x_{2} ;
$$

(2) there exists a continuous map $\phi:[0,+\infty) \rightarrow \mathbb{R}$ such that

$$
\left|X_{2}\left(x_{1}(t), x_{2}(t)\right)\right| \leq \phi(t)
$$

and

$$
\int_{0}^{+\infty} \phi(t) \mathrm{d} t<\infty
$$

then, the system (1.2) is p-partially rationally asymptotically stable.

Proof. We assume that the assumptions of Theorem 2.6 hold, conditions (2.16) and (2.17) imply that $0 \in \mathbb{R}^{n}$ is Lyapunov stable for the closed loop system $\dot{x}=X(x)=\left(X_{1}\left(x_{1}, x_{2}\right), X_{2}\left(x_{1}, x_{2}\right)\right.$ ) (see [65], Thm. 0.4.1, p. 26 for instance).

By combining the assertions (i) and (ii), we obtain constants $c_{3}>0$ and $r_{3}>r_{2}>0$ such that

$$
\dot{V} \leq-c V^{\frac{r_{3}}{r_{2}}}, c=\frac{c_{3}}{c_{2}^{r_{3} / r_{2}}}
$$

Since $r_{3}>r_{2}$, then $\frac{r_{3}}{r_{2}}=1+\alpha$, where $\alpha=\frac{r_{3}-r_{2}}{r_{2}}>0$, then, (2.19) is equivalent to

$$
\dot{V} \leq-c V^{1+\alpha}
$$


the last inequality implies

$$
V \leq \frac{1}{(a t+b)^{\frac{1}{\alpha}}}, \text { where } a=c \alpha, b=\frac{1}{V(x(0))^{\alpha}}
$$

Hence, from (2.16), we get

$$
\left|x_{1}\right| \leq \frac{c_{0}}{(a t+b)^{\gamma}}, c_{0}=c_{1}^{-1 / r_{1}}, \gamma=\frac{1}{r_{1} \alpha}
$$

therefore, the system (1.2) is rationally asymptotically stable with respect to $x_{1}$.

The convergence of the partial state $x_{2}$ follows from Lebesgue dominated convergence theorem. Hence, the system (1.2) is $p$-partially rationally asymptotically stable.

Example 2.7 ([40]). Considering the following system

$$
\left\{\begin{array}{l}
\dot{\omega}_{1}=u_{1} \\
\dot{\omega}_{2}=u_{2} \\
\dot{\omega}_{3}=\omega_{1} \omega_{2} \\
\dot{\omega}_{4}=\left(\omega_{3}+\omega_{2}\right)^{2} \\
\dot{\omega}_{5}=\left(\omega_{1}-\omega_{3}^{2 p}\right)^{\frac{k+p}{k}}
\end{array}\right.
$$

where $k, p \in \mathbb{N}^{*}$ or $k, p$ are two non-negative odd rational numbers, such that $2 p>1$ and $k>p$.

$x=\left(\omega_{1}, \omega_{2}, \omega_{3}, \omega_{4}, \omega_{5}\right)^{\prime} \in \mathbb{R}^{5}$ is the state and $u=\left(u_{1}, u_{2}\right) \in \mathbb{R}^{2}$ is the control. The subsystem

$$
\left\{\begin{array}{l}
\dot{\omega}_{1}=u_{1} \\
\dot{\omega}_{2}=u_{2} \\
\dot{\omega}_{3}=\omega_{1} \omega_{2},
\end{array}\right.
$$

represents the angular velocity control of a rigid spacecraft with only two controllers which has been studied extensively in the literature. The issue of feedback stabilization of the angular velocities has been solved using various approaches [1-4].

We have shown in [40], that if $k>2 p>1$, then, the following Hölder feedback laws

$$
\begin{aligned}
& u_{1}=2 p \omega_{3}^{2 p-1} \omega_{1} \omega_{2}-\left(\omega_{2}+\omega_{3}\right) \omega_{3}^{2 k-1}+\omega_{3}^{2 k}-\alpha_{1}\left(\omega_{1}-\omega_{3}^{2 p}\right)^{1+\frac{2 p}{k}}, \alpha_{1}>1 \\
& u_{2}=-\omega_{1} \omega_{2}-\omega_{3}^{2(k+p)-1}-\alpha_{2}\left(\omega_{2}+\omega_{3}\right)^{1+\frac{2 p}{k}}, \alpha_{2}>1,
\end{aligned}
$$

render the system (2.22) three-rationally partially asymptotically stable.

The rational partial asymptotic stabilizability of the system (2.22) is obtained with the help of the candidate Lyapunov function

$$
V=\frac{1}{2 k} \omega_{3}^{2 k}+\frac{1}{2}\left(\omega_{1}-\omega_{3}^{2 p}\right)^{2}+\frac{1}{2}\left(\omega_{2}+\omega_{3}\right)^{2},
$$

and it was shown that under the feedback laws (2.23), the Lyapunov function $V$ satisfies the differential inequality

$$
\dot{V} \leq-c V^{1+\frac{p}{k}}, c>0 .
$$

Then, Theorem 2.6 permits to conclude.

\section{Finite-time partial stability}

\subsection{Finite-time partial stability of homogeneous systems}

In the following subsection, we will give a similar result to [14], Theorem 7.1, concerning the finite-time partial stability of nonlinear control system by homogeneous feedback laws. 
Theorem 3.1. Let the control system

$$
\dot{x}_{1}=X_{1}\left(x_{1}, x_{2}, u\right), \dot{x}_{2}=X_{2}\left(x_{1}, x_{2}, u\right) .
$$

If there exists a $C^{0}$-homogeneous feedback law $u\left(x_{1}, x_{2}\right)$, satisfying $u\left(0, x_{2}\right)=0$, such that in closed loop:

(1) the system (3.1) is p-partially asymptotically stable;

(2) the system (3.1) is homogeneous of negative degree $m$ with respect to appropriate dilation;

(3) $X_{2}\left(0, x_{2}, 0\right)=0$.

Then (3.1) is p-partially stable in finite-time.

Proof. We assume that there exists a $C^{0}$-homogeneous feedback law $u\left(x_{1}, x_{2}\right)$, satisfying $u\left(0, x_{2}\right)=0$, such that in closed loop, the system (3.1) is p-partially asymptotically stable. By using inverse Lyapunov function for the partial asymptotic stability [36,67], the condition (1) implies that there exists a continuous, positive-definite function $V$ with respect to $x_{1}$ such that $\dot{V}$ is negative-definite with respect to $x_{1}$. Also by using [14], Theorem 6.2 (p. 117), the function $V$ is homogeneous of degree $l$ and $\dot{V}$ is homogeneous of degree $l+m$; see, for instance, [65], Theorem 2.5.1. By using [14], Theorem 6.2, the closed loop system (3.1) is finite-time stable with respect to $x_{1}$.

From the definition of finite-time stability, there exists a settling time $T>0$, such that $x_{1}(t)=0$ for all $t \geq T$. Then, from assumption (3) we have

$$
X_{2}\left(x_{1}(t), x_{2}(t), u\left(x_{1}, x_{2}\right)\right)=X_{2}\left(0, x_{2}(t), 0\right)=0, \forall t \geq T
$$

and

in this case we get

$$
\int_{T}^{+\infty}\left|X_{2}\left(x_{1}(t), x_{2}(t), u\left(x_{1}, x_{2}\right)\right)\right| \mathrm{d} t=\int_{T}^{+\infty} 0 \mathrm{~d} t=0
$$

$$
\int_{0}^{+\infty}\left|X_{2}\left(x_{1}(t), x_{2}(t), u\left(x_{1}, x_{2}\right)\right)\right| \mathrm{d} t=\int_{0}^{T}\left|X_{2}\left(x_{1}(t), x_{2}(t), u\left(x_{1}, x_{2}\right)\right)\right| \mathrm{d} t<\infty
$$

and then

$$
x_{2}(t)=x_{2}(0)+\int_{0}^{T} X_{2}\left(x_{1}(s), x_{2}(s), u\left(x_{1}, x_{2}\right)\right) \mathrm{d} s<\infty,
$$

which implies $x_{2}(t)$ is constant for $t \geq T$.

In the sequel we will give another alternative to stabilize the system describing the unicycle. This alternative is based on finite-time partial stabilization by homogeneous feedback law of negative degree (Thm. 3.1), and on Bhat and Bernstein feedback law [11].

Proposition 3.2. Let be $\alpha \in(0,1)$, and let the feedback:

$$
u=-\operatorname{sgn}\left(x_{2}\right)\left|x_{2}\right|^{\alpha}-\operatorname{sgn}\left(x_{1}\right)\left|x_{1}\right|^{\frac{\alpha}{2-\alpha}} .
$$

Then $(0,0) \in \mathbb{R}^{2} \times \mathbb{R}$ is 2-partially stable in finite-time for the closed-loop system (2.2) with $v_{1}$ and $v_{2}$ defined by

$$
\begin{aligned}
& v_{1}=\left|x_{1}\right|^{\alpha_{1}}+\left|x_{2}\right|^{\alpha_{1}(2-\alpha)}, \text { where } 0<\alpha_{1}<\frac{1-\alpha}{2-\alpha} \\
& \text { and } v_{2}:=u v_{1} .
\end{aligned}
$$


Proof. Let the candidate Lyapunov function be defined as in [11]:

$$
V=\frac{1}{2} x_{2}^{2}+\frac{2-\alpha}{2}\left|x_{1}\right|^{\frac{2}{2-\alpha}} .
$$

The time derivative of $V$ along the system (2.2) is given by

$$
\dot{V}=x_{2} v_{2}+\left|x_{1}\right|^{\frac{\alpha}{2-\alpha}} x_{2} \operatorname{sgn}\left(x_{1}\right) v_{1},
$$

with the help of our feedbacks (3.2) and (3.3), $\dot{V}$ becomes

$$
\dot{V}=-\left(\left|x_{1}\right|^{\alpha}+\left|x_{2}\right|^{\alpha_{1}(2-\alpha)}\right)\left|x_{2}\right|^{1+\alpha} \leq-\left|x_{2}\right|^{1+2 \alpha_{1}+\alpha\left(1-\alpha_{1}\right)} \leq 0 .
$$

Then the manifold $\left\{\left(x_{1}, x_{2}\right): \dot{V}=0\right\}$ is equivalent to $\left\{\left(x_{1}, x_{2}\right): x_{2}=0\right\}$.

If we have $\forall t \geq 0, x_{2}(t)=0$, then $\dot{x}_{2}(t)=0$ and therefore we get:

$$
-\operatorname{sgn}\left(x_{1}\right)\left|x_{1}\right|^{\frac{\alpha}{2-\alpha}+\alpha_{1}}=0 \Leftrightarrow x_{1}=0 .
$$

We conclude, by LaSalle's theorem, that the only invariant set in the sub-manifold $\left\{\left(x_{1}, x_{2}\right): \dot{V}=0\right\}$ is the origin $(0,0) \in \mathbb{R}^{2}$, and therefore the closed loop system is 2 -asymptotically stable.

In closed loop, the subsystem described by $x_{1}$ and $x_{2}$ is given as follows:

$$
\left\{\begin{array}{l}
\dot{x}_{1}=f_{1}\left(x_{1}, x_{2}\right)=x_{2}\left(\left|x_{1}\right|^{\alpha_{1}}+\left|x_{2}\right|^{\alpha_{1}(2-\alpha)}\right) \\
\dot{x}_{2}=f_{2}\left(x_{1}, x_{2}\right)=\left(\left|x_{1}\right|^{\alpha_{1}}+\left|x_{2}\right|^{\alpha_{1}(2-\alpha)}\right)\left(-\operatorname{sgn}\left(x_{2}\right)\left|x_{2}\right|^{\alpha}-\operatorname{sgn}\left(x_{1}\right)\left|x_{1}\right|^{\frac{\alpha}{2-\alpha}}\right) .
\end{array}\right.
$$

Let the dilation $\Delta_{k}\left(x_{1}, x_{2}\right)=\left(k^{2-\alpha} x_{1}, k x_{2}\right)$ where $k>0$.

Simple calculation yields

$$
\begin{aligned}
f_{1}\left(\Delta_{k}\left(x_{1}, x_{2}\right)\right) & =k^{1+\alpha_{1}(2-\alpha)} f_{1}\left(x_{1}, x_{2}\right) \\
& =k^{2-\alpha+\beta} f_{1}\left(x_{1}, x_{2}\right)
\end{aligned}
$$

and

$$
\begin{aligned}
f_{2}\left(\Delta_{k}\left(x_{1}, x_{2}\right)\right) & =k^{\alpha_{1}(2-\alpha)+\alpha} f_{2}\left(x_{1}, x_{2}\right) \\
& =k^{1+\beta} f_{2}\left(x_{1}, x_{2}\right)
\end{aligned}
$$

where

Since

$$
\beta=1+\left(\alpha_{1}-1\right)(2-\alpha) .
$$

then

$$
0<\alpha_{1}<\frac{1-\alpha}{2-\alpha}
$$

and

$$
\left(\alpha_{1}-1\right)(2-\alpha)<-1,
$$

$$
\beta<0 .
$$

Hence, we conclude that the vector field $f=\left(f_{1}, f_{2}\right)$ is homogeneous of negative degree $\beta$ with respect to the dilation $\Delta_{k}\left(x_{1}, x_{2}\right)=\left(k^{2-\alpha} x_{1}, k x_{2}\right)$. From Theorem 3.1, the system (3.7) is 2-finite-time stable.

Since the feedback controller $u_{1}$ given in (3.3) satisfies the condition

$$
v_{1}\left((0,0), x_{3}\right)=0,
$$

then, in virtue to condition 3 of Theorem 3.1 the state $x_{3}(t)$ is constant for $t$ large enough. 


\subsection{Continuous finite-time stabilizing feedbacks of the unicycle}

Based on the pioneer work of Bhat and Bernstein [12], we show also how to stabilize in finite-time the system (1.1) with respect to $\left(x_{1}, x_{2}\right)$, while the state $x_{3}$ converges in finite-time. These feedback controllers are also homogeneous with negative degree with respect to dilation $\Delta_{\varepsilon}\left(x_{1}, x_{2}\right)=\left(\varepsilon^{2-\alpha} x_{1}, \varepsilon x_{2}\right)$ where $\varepsilon>0$ and $\alpha \in(0,1)$. The construction proof reposed on [12], Theorem 1, and uses the Lyapunov direct method.

Proposition 3.3 ([37]). Let be $\alpha \in(0,1)$, and let the feedback

$$
u=-\operatorname{sgn}\left(x_{2}\right)\left|x_{2}\right|^{\alpha}-\operatorname{sgn}\left(\phi\left(x_{1}, z_{2}\right)\right)\left|\phi\left(x_{1}, x_{2}\right)\right|^{\frac{\alpha}{2-\alpha}},
$$

where

$$
\phi\left(x_{1}, x_{2}\right)=x_{1}+\frac{1}{2-\alpha} \operatorname{sgn}\left(x_{2}\right)\left|x_{2}\right|^{2-\alpha} .
$$

Let $r, s>0$ be two real-numbers and let the candidate Lyapunov function

$$
V\left(x_{1}, x_{2}\right)=\frac{2-\alpha}{3-\alpha}\left|\phi\left(x_{1}, x_{2}\right)\right|^{\frac{3-\alpha}{2-\alpha}}+s x_{2} \phi\left(x_{1}, x_{2}\right)+\frac{r}{3-\alpha}\left|x_{2}\right|^{3-\alpha} .
$$

Then $(0,0) \in \mathbb{R}^{2} \times \mathbb{R}$ is 2-partially stable in finite-time for the closed loop system (2.2) with $v_{1}$ and $v_{2}$ defined by

such that

$$
v_{1}(x):=\left(V\left(x_{1}, x_{2}\right)\right)^{\beta}, \beta>0,
$$

$$
\gamma:=\beta+\frac{2}{3-\alpha}<1, \text { and } v_{2}:=v_{1} u
$$

Proof. The time-derivative of $V$ along the system (2.2) is given by

$$
\dot{V}=\frac{\partial V}{\partial x_{1}} x_{2} v_{1}+\frac{\partial V}{\partial x_{2}} u v_{1}=\left.v_{1} \dot{V}\right|_{(3.12)},
$$

where $\left.\dot{V}\right|_{(3.12)}$ denotes the time derivative of $V$ for the following system

$$
\dot{x}_{1}=x_{2}, \dot{x}_{2}=u \text {. }
$$

Thanks to [12], Proposition $1,\left.\dot{V}\right|_{(3.12)}$ satisfies for all $\left(x_{1}, x_{2}\right) \in \mathbb{R}^{2}$ the inequality

$$
\left.\dot{V}\right|_{(3.12)} \leq-c V^{\frac{2}{3-\alpha}}, c>0
$$

Then, if we choose the feedback $v_{1} \geq 0$, the inequality (3.11) becomes

$$
\dot{V} \leq-c v_{1} V^{\frac{2}{3-\alpha}}
$$

Then one can take

$$
v_{1}=V^{\beta}, \text { where } \beta>0 \text { such that } \gamma:=\beta+\frac{2}{3-\alpha} \in(0,1)
$$

With this $v_{1}$, we get

$$
\dot{V} \leq-c V^{\gamma} \leq 0
$$

which, together with Theorem 3.1, condition (3) (see also [37]), concludes the proof of Proposition 3.3.

Remark 3.4. In (3.14), if we choose $\beta=\frac{1-\alpha}{3-\alpha}$, then we get $\dot{V} \leq-c V$ and therefore, in closed-loop, (1.1) is 2-exponentially stable. Convergence of the state $x_{3}$ follows from Lebesgue convergence dominates theorem. 
The partial finite-time stabilizing controllers in the previous section were unbounded, and these feedback laws cannot give any information about the seize of the convergence of the "uncontrolled part" $x_{3}$. To this end, we give a "measure" of the seize of the convergence of the state $x_{3}$. We prove that there exist stabilizing feedback laws that make the considered system two-partially stable in finite-time and for all $\varepsilon>0$, such that $|x(0)| \leq \frac{\varepsilon}{2}$, then the limit $l$ of the state $x_{3}$ satisfies $|l| \leq \varepsilon$.

Given a positive number $\varepsilon$, we define the saturation function as

$$
\begin{aligned}
\operatorname{sat}_{\varepsilon}(x): & =x, \text { if }|x|<\varepsilon \\
& =\varepsilon \operatorname{sgn}(x), \text { if }|x| \geq \varepsilon,
\end{aligned}
$$

clearly, $\left|\operatorname{sat}_{\varepsilon}(x)\right| \leq \varepsilon$ for every $x \in \mathbb{R}$.

Now, we are ready to state our result.

Proposition 3.5. Let $\varepsilon>0$, and $T>0$ be a real number supposed to be large enough. Let the feedback $u$ and the Lyapunov function $V$ introduced in Proposition 3.3. We assume that $|x(0)| \leq \varepsilon / 2$, where $x=\left(x_{1}, x_{2}, x_{3}\right)^{\prime}$. by

Then $(0,0) \in \mathbb{R}^{2} \times \mathbb{R}$ is 2-partially stable in finite-time for the closed loop system (2.2) with $v_{1}$ and $v_{2}$ defined

$$
v_{1}(x):=\operatorname{sat}_{\varepsilon_{0}}\left(V^{\beta}\right) \text {, where } \varepsilon_{0}=\frac{\varepsilon}{2 T} \text {, and } \beta>0, \text { such that } \gamma:=\beta+\frac{2}{3-\alpha}<1,
$$

and

Therefore

$$
v_{2}:=v_{1} u
$$

$$
\left|x_{3}(t)\right| \leq \varepsilon, \forall t \geq 0 .
$$

Proof. Clearly, under the bounded feedback (3.15) and from Proposition 3.3, the system (2.2) is two-partially stable in finite-time. Indeed:

- if $V\left(x_{1}, x_{2}\right) \leq\left(\varepsilon_{0}\right)^{1 / \beta}$, then $\operatorname{sat}_{\varepsilon_{0}}\left\{\left(V\left(x_{1}, x_{2}\right)\right)^{\beta}\right\}=\left(V\left(x_{1}, x_{2}\right)\right)^{\beta}$, in this case it follows from Proposition 3.3 that (2.2) is two-partially stable in finite-time;

- if $V\left(x_{1}, x_{2}\right) \geq\left(\varepsilon_{0}\right)^{1 / \beta}$, then $\operatorname{sat}_{\varepsilon_{0}}\left\{\left(V\left(x_{1}, x_{2}\right)\right)^{\beta}\right\}=\varepsilon_{0}$, in this case, from (3.13), we get

$$
\dot{V} \leq-c \varepsilon_{0} V^{\frac{2}{3-\alpha}} .
$$

Since $\frac{2}{3-\alpha} \in(0,1)$, then, in closed loop, the system $(2.2)$ is two-partially stable in finite-time.

Therefore, there exits a finite-time $T_{0}$ such that $x_{1}(t)=x_{2}(t)=0, \forall t \geq T_{0}$.

By integration, the state $x_{3}$ verifies $x_{3}(t)=x_{3}(0)+\int_{0}^{T_{0}} v_{1}(x(s)) \mathrm{d} s$, and, since $v_{1} \leq \frac{\varepsilon}{2 T}$, then, by triangular inequality we get

$$
\left|x_{3}(t)\right| \leq\left|x_{3}(0)\right|+T_{0} \frac{\varepsilon}{2 T} .
$$

Since $T$ is large enough, then $\frac{T_{0}}{T} \leq 1$, and, from the assumption $|x(0)| \leq \frac{\varepsilon}{2}$ and (3.17), we get

$$
\left|x_{3}(t)\right| \leq \frac{\varepsilon}{2}+\frac{\varepsilon}{2}=\varepsilon, \forall t \geq 0 .
$$

Hence, the state $x_{3}$ is constant for $t$ large enough, then the limit of $x_{3}$ denoted by $l(x(0))$ satisfies

$$
|l(x(0))| \leq \varepsilon .
$$



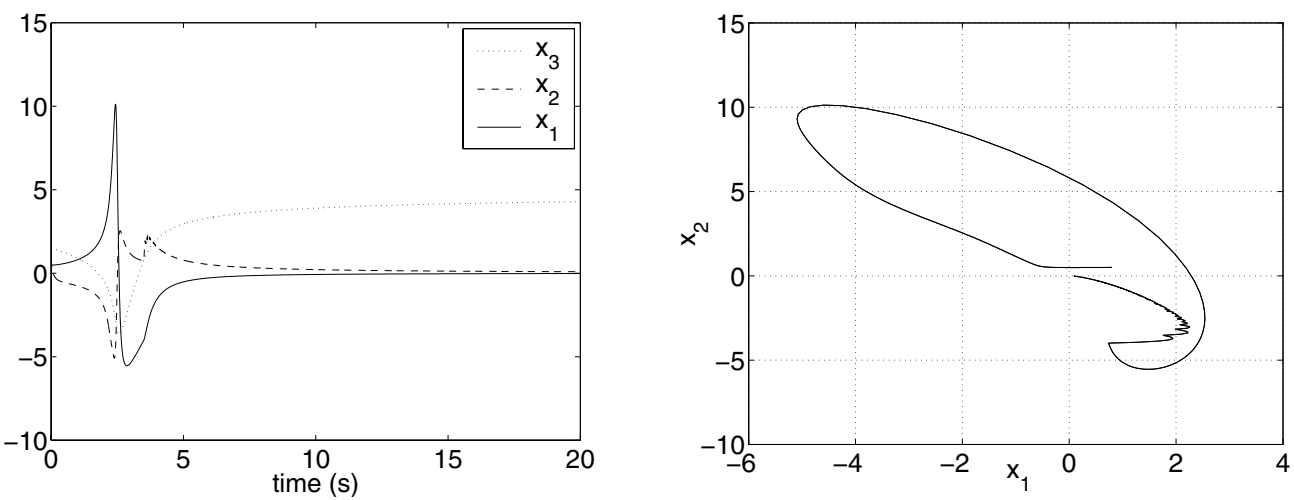

Figure 2. Trajectories of the state $x_{1}, x_{2}$ and $x_{3}$ (left); motion in the plane $\left(x_{1}, x_{2}\right)$ (right).
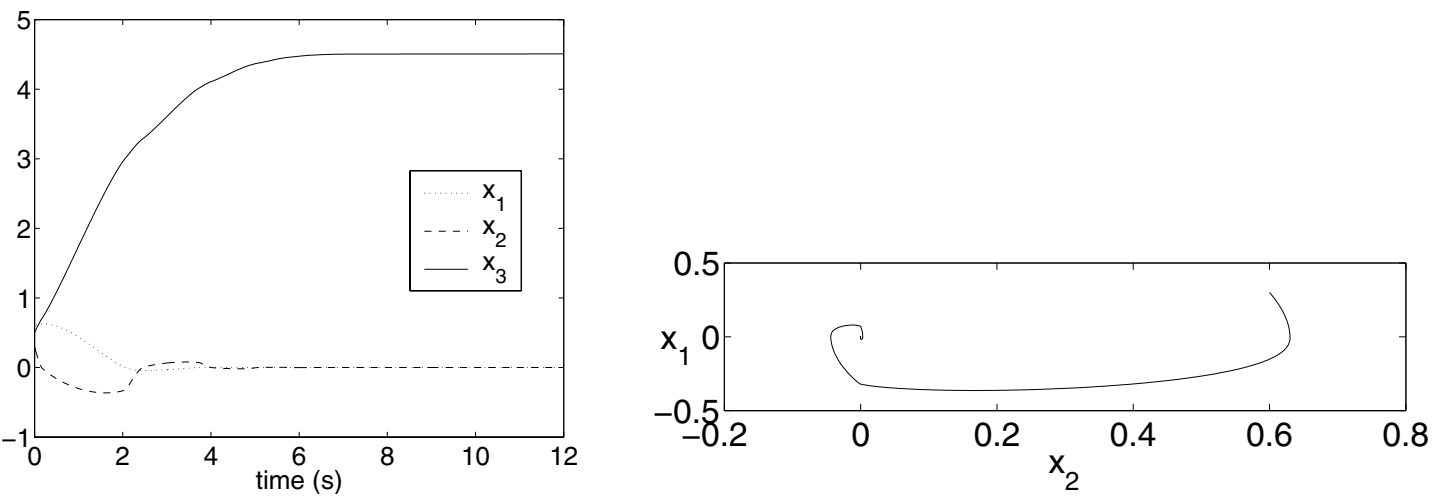

Figure 3. Trajectories of the state $x_{1}, x_{2}$ and $x_{3}$ (left); motion in the plane $\left(x_{2}, x_{1}\right)$ (right).

Remark 3.6. The conclusion of Proposition 3.5 still hold if we replace the feedback $v_{1}$ in (3.15) by the following $v_{1}=\operatorname{sat}_{\varepsilon_{0}}\left\{\left|x_{1}\right|^{\alpha_{1}}+\left|x_{2}\right|^{\alpha_{1}(2-\alpha)}\right\}$ where $0<\alpha_{1}<\frac{1-\alpha}{2-\alpha}$.

\subsection{Numerical simulations}

In the following charts we present numerical simulations to validate our feedback laws constructed by the two approaches. These simulations show how the system (2.2) is two-partially asymptotically stabilizable.

Approach 1: Asymptotic estimation is presented in Figure 2.

Approach 2: Homogeneous finite-time stabilizing feedbacks is presented in Figure 3.

Approach 3: Continuous finite-time stabilizing feedbacks is verified in Figure 4.

The boundedness of the limit of $x_{3}$ is obtained with the initial condition $x_{0}=(1.5,0.8,0.5)$ and with the saturation $\varepsilon / 2 T=20 / 120(\varepsilon=20$ and $T=60)$. The simulation shows that $|l| \leq 7.16$ where $l$ is the finite-time limit of $x_{3}$.

\section{Robust stabilization}

Ever since periods ago, robust stabilization of nonlinear control systems has received a lot of attention by the community of control theory; see [10,32-34,44,45,54,62,63], and the references therein. In [10], the authors 

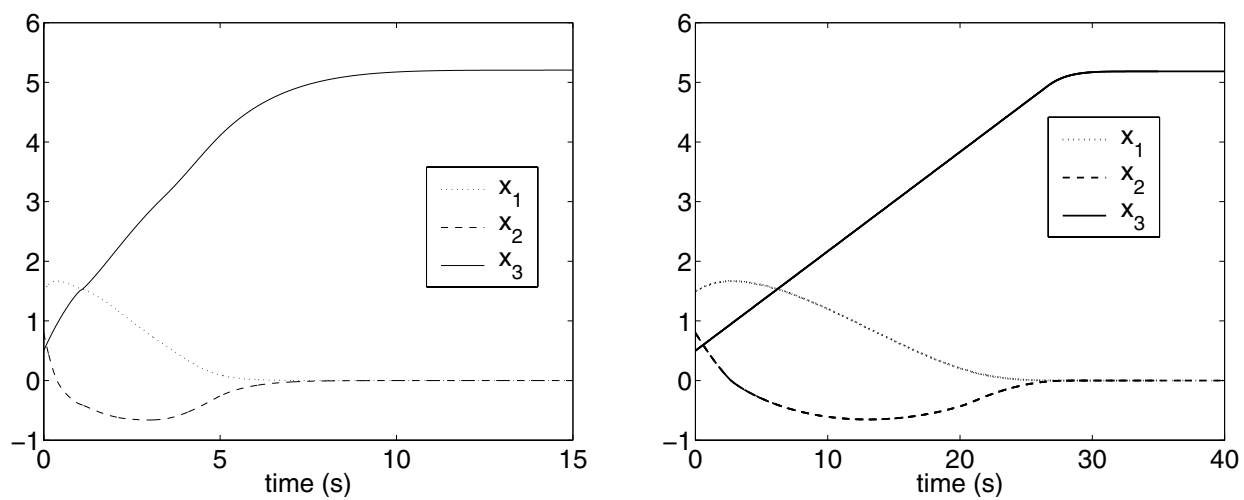

Figure 4. Trajectories of the state $x_{1}, x_{2}$ and $x_{3}$ (left); trajectories of the state $x_{1}, x_{2}$ and boundedness the limit of the state $x_{3}$ (right).

have shown that all flat chained systems can be stabilized by hybrid controls to obtain robustness properties. The method is based essentially on iterations of chosen open-loop steering controls. It was shown in [44] that no static continuous homogeneous exponential stabilizer can be robust for all driftless control systems. In this context, the authors established sufficient conditions for the non-robustness of homogeneous stabilizers like a loss of rank of the accessibility condition. In [45], the authors have constructed Lipschitz-continuous time-varying feedback laws which make all chained systems locally robustly asymptotically stable with respect to unmodelled dynamics.

One question can be asked: Do the continuous feedback laws established for the partial finite-time stability of the nominal Brockett's system are robustly with respect to small disturbance affect the system?

We consider the perturbed system generated by

$$
\begin{aligned}
& \dot{y}_{1}=u_{1} \cos \theta+\varepsilon u_{1} \sin \theta \\
& \dot{y}_{2}=u_{1} \sin \theta-\varepsilon u_{1} \cos \theta \\
& \dot{\theta}=u_{2},
\end{aligned}
$$

where $\varepsilon$ is a real parameter such that $|\varepsilon| \ll 1$. The state is given by $y=\left(y_{1}, y_{2}, \theta\right)^{\prime} \in \mathbb{R}^{3}$ and $u=\left(u_{1}, u_{2}\right) \in \mathbb{R}^{2}$ is the vector control. Clearly the case $\varepsilon=0$ corresponds to the unicycle system. When $\varepsilon$ is small enough, the terms $\cos \theta+\varepsilon \sin \theta$ and $\sin \theta-\varepsilon \cos \theta$ can be seen (by transformation of $a_{1} \cos b+a_{2} \sin b$ ) respectively as $\cos (\theta-\varepsilon)$ and $\sin (\theta-\varepsilon)$, in this case, the system (4.1) becomes

$$
\begin{aligned}
& \dot{y}_{1}=u_{1} \cos (\theta-\varepsilon) \\
& \dot{y}_{2}=u_{1} \sin (\theta-\varepsilon) \\
& \dot{\theta}=u_{2},
\end{aligned}
$$

then, the perturbation in the system (4.2) can be interpreted as a measurement error from the angle, where $\varepsilon$ is a small bias in the orientation, see [50] for more detail on this subject. In the next, we show that the perturbed system (4.1) can be stabilized partially and locally in finite-time.

\subsection{Existence of the stabilizing feedback law of (4.1)}

Under the new coordinates introduced in (2.1), the system (4.1) can be written in the form

$$
\begin{aligned}
& \dot{x}_{1}=x_{2} v_{1}+\varepsilon\left(v_{2}+x_{1} v_{1}\right) \\
& \dot{x}_{2}=v_{2} \\
& \dot{x}_{3}=v_{1} .
\end{aligned}
$$


Note that $\left(y_{1}, y_{2}\right)=0$ is equivalent to $\left(x_{1}, x_{2}\right)=0$. It is clear that the parameterized system (4.3) cannot be stabilized by continuous state feedback laws, since all points of the form $(\varepsilon, 0,0)^{\prime}, \varepsilon \neq 0$ are not in $f\left(\mathbb{R}^{3} \times \mathbb{R}^{2}\right)$, where $f(x, u)=\left(x_{2} v_{1}+\varepsilon\left(v_{2}+x_{1} v_{1}\right), v_{2}, v_{1}\right)^{\prime}$. Also, we note that the global controllability propriety of (4.3) is easily checked by using Chow's theorem.

So, the stabilization problem should be treated in the finite-time partial stabilization sense. The goal of this subsection is to prove the existence of stabilizing feedback that renders the system (4.3) two-partially stable in finite-time. We rewrite the input $v_{2}$ in the form $v_{2}=u v_{1}$, where $u$ and $v_{1}$ are two suitable feedback laws to be constructed.

The system (4.3) becomes

$$
\begin{aligned}
& \dot{x}_{1}=\left(x_{2}+\epsilon\left(u+x_{1}\right)\right) v_{1} \\
& \dot{x}_{2}=u v_{1} \\
& \dot{x}_{3}=v_{1} .
\end{aligned}
$$

Let the linear parameterized subsystem

$$
\begin{aligned}
& \dot{x}_{1}=x_{2}+\epsilon\left(u+x_{1}\right) \\
& \dot{x}_{2}=u .
\end{aligned}
$$

The key construction of the stabilizing feedback law of (4.3) is given in the following lemmas.

Lemma 4.1. For all $\epsilon$, the system (4.4) is globally finite-time stabilizable.

Proof. The linear system (4.4) takes the form $\dot{z}=A z+B u$, where $z=\left(x_{1}, x_{2}\right)$,

$$
A=\left(\begin{array}{cc}
\epsilon & 1 \\
0 & 0
\end{array}\right)
$$

and

$$
B=\left(\begin{array}{c}
\epsilon \\
1
\end{array}\right)
$$

It is clear that the Kalman controllability condition is satisfied; since $\forall \epsilon \in \mathbb{R}, \operatorname{span}(B, A B)=\mathbb{R}^{2}$, therefore the system (4.4) is controllable. By using Bhat and Bernstein theorem (see [14], Thm. 8.1, p. 123), it means that every controllable linear system is globally finite-time stable. Then the system (4.4) is globally finite-time stable through continuous state feedback.

Lemma 4.2. For all $\epsilon$, the system (4.3) is 2-partially globally finite-time stabilizable.

Proof. Since the linear control system (4.4) is globally finite-time stabilizable, therefore the settling-time is a continuous function, then by converse Lyapunov theorem due to Bhat and Bernstein [13], Theorem 4.3 (p. 761), there exists for (4.4) a continuous feedback law $u(x)$, a $C^{1}$ candidate Lyapunov function $V_{\epsilon}$, a constant $c_{\epsilon}>0$ and a real $\alpha \in(0,1)$ such that in closed loop we have $\dot{V}_{\epsilon} \leq-c_{\epsilon} V_{\epsilon}^{\alpha}$.

The time derivative of $V_{\epsilon}$ along the system (4.3), and taking into account the decomposition $v_{2}=u v_{1}$, then we get

$$
\dot{V}_{\epsilon}=\left.v_{1} \dot{V}_{\epsilon}\right|_{(4.4)}
$$

where $\left.\dot{V}_{\epsilon}\right|_{(4.4)}$ is the time derivative of $V$ along (4.4). In this case, we can choose $v_{1}=V_{\epsilon}^{\beta}$, where $\beta>0$ and $\beta+\alpha \in(0,1)$, and finally we conclude by [12], Proposition 1 .

\subsection{Robust stabilization of Brockett's integrator}

In this section we address the problem of robust feedback stabilization of the nonlinear Brockett's integrator. We will ask if the stabilizing feedback laws proposed in Proposition 3.2 also stabilize locally partially and in finite-time the perturbed system (4.3). Before giving the open question we recall the following definition. 
Definition 4.3 ([44]). An admissible perturbation is a smooth mapping $\varepsilon \mapsto g(\varepsilon,$.$) such that for every \varepsilon \in \mathbb{R}$, $g(\varepsilon,$.$) is a smooth vector field in \mathbb{R}^{n}$ and $g(\varepsilon, 0)=0$ for all $x \in \mathbb{R}^{n}$.

Inspired by the definition given in [44], we can define:

Definition 4.4. A continuous feedback law $u: \mathbb{R}^{p} \times \mathbb{R}^{n-p} \ni x=\left(x_{1}, x_{2}\right) \mapsto u(x) \in \mathbb{R}^{m}$ making the origin of the nominal system $\dot{x}=X_{0}(x, u) p$-partially stable in finite-time is robust with respect to unmodelled dynamics $\dot{x}=X_{\varepsilon}(x, u):=X_{0}(x, u)+g(\varepsilon, x)$, for $\varepsilon \in \mathbb{R}$, where $g(\varepsilon,$.$) is an admissible perturbation, if there exists \varepsilon_{0}>0$ such that, for $|\varepsilon| \leq \varepsilon_{0}, u$ also makes the origin of $\dot{x}=X_{\varepsilon}(x, u) p$-partially stable in finite-time.

A possible key to study the robustness control is the following lemma:

Lemma 4.5. Consider in $\mathbb{R}^{p} \times \mathbb{R}^{n-p}$ the continuous system in the following form

$$
\dot{x}_{1}=X_{1}\left(x_{1}, x_{2}\right)+\widehat{X_{1}}\left(x_{1}, x_{2}\right), \dot{x}_{2}=X_{2}\left(x_{1}, x_{2}\right)+\widehat{X_{2}}\left(x_{1}, x_{2}\right) .
$$

We assume that:

(1) $X_{i}\left(0, x_{2}\right)=\widehat{X_{i}}\left(0, x_{2}\right)=0$, for $i=1,2$;

(2) $X_{1}$ is homogeneous of negative degree $m$ with respect to appropriate dilation $\left(r_{1}, \ldots, r_{n}\right)^{\prime}$;

(3) the system $\dot{x}_{1}=X_{1}\left(x_{1}, x_{2}\right), \dot{x}_{2}=X_{2}\left(x_{1}, x_{2}\right)$ is p-partially asymptotically stable;

(4)

$$
\lim _{k \rightarrow 0^{+}} \frac{\widehat{X_{j}}\left(k_{1}^{r} x_{1}, k_{2}^{r} x_{2}, \ldots, k_{n}^{r} x_{n}\right)}{k^{m+r_{i}}}=0, j=1,2, i=1, \ldots, n,
$$

uniformly on the unity sphere $\mathbb{S}^{n-1}$ of $\mathbb{R}^{n}$.

Then (4.6) is p-locally partially stable in finite-time.

Remark 4.6. This lemma is firstly given in [34] in the particular case $p=n$. As cited in [34], the proof reposed in part on [12], Theorem 1 or [37], Proposition 1 and on (iii) of Theorem 3.1.

Now, we are ready to give the open question.

Open question: Is the feedbacks proposed in Proposition 3.2 also locally partially stabilize in finite-time the perturbed system (4.3)?

\section{Finite-Time PARTial STABILITy By Discontinuous STABILIZING FEEDBACK LAWS}

Dynamical control systems described by differential equations with discontinuous right-hand sides are the subject of numerous papers and books; see [27,51,52]. From practical point of view, such systems do arise and require analysis. Examples of such systems are mechanical systems with Coulomb friction modeled as a force proportional to the sign of a velocity, and systems whose control laws have discontinuities; see $[51,52]$.

To this end, this section is devoted to study the possible case to stabilize the Brockett's integrator partially in finite-time by discontinuous feedback laws. These feedback laws are inspired by [51], Theorem 4.4 (p. 96). Before the construction of the discontinuous stabilizing feedbacks, we define the notion of partial stability in finite time by discontinuous feedback laws.

Throughout, the precise meaning of the differential equation $\dot{x}=X(x)$ with a piecewise continuous right-hand side is defined in the sense of Filippov (see for instance $[27,51]$ ).

Definition 5.1 ( $p$-finite-time partial stability). Let $X: \mathbb{R}^{p} \times \mathbb{R}^{n-p} \cong \mathbb{R}^{n} \rightarrow \mathbb{R}^{n}, x=\left(x_{1}, x_{2}\right) \in \mathbb{R}^{p} \times \mathbb{R}^{n-p} \mapsto$ $X(x) \in \mathbb{R}^{n}$, be defined and discontinuous on a neighborhood of $(0,0) \in \mathbb{R}^{p} \times \mathbb{R}^{n-p}$. We assume that

$$
X\left(0, x_{2}\right)=0 .
$$


One says that $(0,0) \in \mathbb{R}^{p} \times \mathbb{R}^{n-p}$ is $p$-partially stable in finite time for $\dot{x}=X(x)$ if:

- $(0,0)$ is Lyapunov stable for $\dot{x}=X(x)$;

- there exist $r>0$ and $T=T(x(0))>0$ called settling-time function, such that, if $\dot{x}=X(x)$ and $|x(0)|<r$, then $x_{1}(t)=0$ for every $t \geq T$ (and therefore $t \mapsto x_{2}(t)$ is constant for $t \geq T$ ).

The control system $\dot{x}=X(x, u)$ is $p$-partially stabilizable in finite-time by discontinuous feedback law, if there exists a discontinuous map $u: \mathbb{R}^{m} \rightarrow \mathbb{R}^{n}$ such that for every $x_{2} \in \mathbb{R}^{n-p}, u\left(0, x_{2}\right)=0$, and such that $(0,0) \in \mathbb{R}^{p} \times \mathbb{R}^{n-p}$ is $p$-partially stable in finite-time for the closed loop system $\dot{x}=X(x, u(x))$.

The following proposition gives the discontinuous feedback stabilizing the unicycle (2.2).

Proposition 5.2. Let be $\alpha \in(0,1)$, and let the feedback:

$$
u=-a \operatorname{sgn}\left(x_{1}\right)-b \operatorname{sgn}\left(x_{2}\right),
$$

if the parameters $a>b>0$. Then $(0,0) \in \mathbb{R}^{2} \times \mathbb{R}$ is 2-partially stable in finite-time for the closed-loop system (2.2) with $v_{1}$ and $v_{2}$ defined by

$$
v_{1}=\left|x_{1}\right|^{\alpha / 2}+\left|x_{2}\right|^{\alpha} \text { and } v_{2}:=u v_{1} .
$$

Proof. The proof of this result is similar to Proposition 3.2 and uses a result due to Orlov [51], Theorem 4.4 (p. 96). Indeed, we assume that the assumptions of the proposition are hold. Let the candidate Lyapunov function defined as

$$
V:=a\left|x_{1}\right|+\frac{1}{2} x_{2}^{2}
$$

A straightforward computations show that

$$
\dot{V}=-b\left|x_{2}\right|\left(\left|x_{1}\right|^{\alpha / 2}+\left|x_{2}\right|^{\alpha}\right):=-V_{1}\left(x_{1}, x_{2}\right) \leq 0,
$$

it is clear that $V_{1}$ is positive semi-definite with respect to $x_{2}$. Let the function $W$ be defined by

$$
W=x_{1} x_{2},
$$

the time derivative of $W$ along the system (2.2) is given by

$$
\dot{W}=\left(x_{2}^{2}-x_{1} u(x)\right) v_{1}(x)=\left(x_{2}^{2}-a\left|x_{1}\right|-b x_{1} \operatorname{sgn}\left(x_{2}\right)\right) v_{1}(x),
$$

within the set $S=\left\{\left(x_{1}, x_{2}\right): b\left|x_{2}\right| \leq \delta\right\}$, we have $x_{2}^{2} \leq \frac{\delta}{b}\left|x_{2}\right|$ and $-b x_{1} \operatorname{sgn}\left(x_{2}\right) v_{1}(x) \leq b\left|x_{1}\right| v_{1}(x)$.

Therefore, we get on the set $S$,

$$
\dot{W} \leq \frac{\delta}{b^{2}} V_{1}\left(x_{1}, x_{2}\right)-(a-b)\left|x_{1}\right| v_{1}\left(x_{1}, x_{2}\right)
$$

Let

$$
W_{1}\left(x_{1}, x_{2}\right)=(a-b)\left|x_{1}\right| v_{1}\left(x_{1}, x_{2}\right),
$$

since $V_{1}\left(x_{1}, x_{2}\right)+W_{1}\left(x_{1}, x_{2}\right)=\left(b\left|x_{2}\right|+(a-b)\left|x_{1}\right|\right) v_{1}\left(x_{1}, x_{2}\right)$ is positive definite, then [51], Theorem 3.3 (p. 55), proves to be applicable to the autonomous system (2.2). Hence the origin is a global asymptotically stable with respect to $\left(x_{1}, x_{2}\right)$.

Let the dilation $\Delta_{k}(x)=\left(k^{2} x_{1}, k x_{2}\right)$ and let $f_{1}(x)=x_{2} v_{1}(x)$ and $f_{2}(x)=u(x) v_{1}(x)$.

A simple calculation shows that

$$
\begin{aligned}
& f_{1}\left(\Delta_{k}(x)\right)=k^{2+\beta} f_{1}(x), \\
& f_{2}\left(\Delta_{k}(x)\right)=k^{1+\beta} f_{2}(x),
\end{aligned}
$$



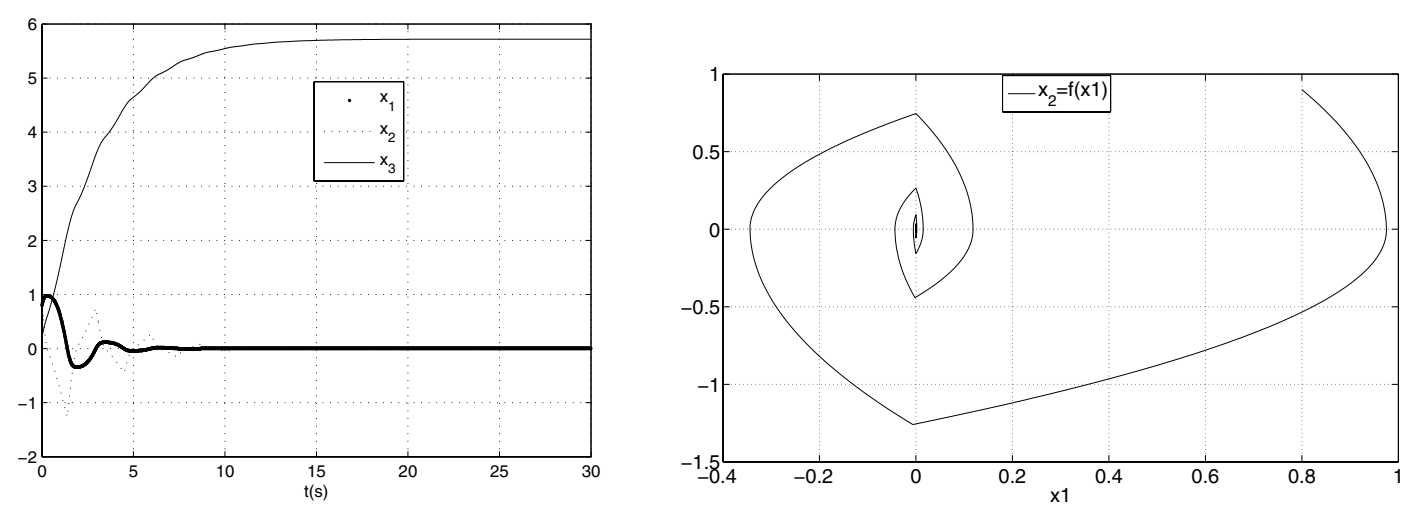

Figure 5. Trajectories of the state $x_{1}, x_{2}$ and $x_{3}$ (left); motion in the plane $\left(x_{1}, x_{2}\right)$ (right).

where $\beta=\alpha-1<0$. Then, the system (1.2) is homogeneous of negative degree with respect to $\Delta_{k}(x)$. According to [51], Theorem 4.1 (p. 92), the system (1.2) is 2-finite-time stable by discontinuous feedback law, and therefore the state $x_{3}(t)$ is constant for a large time $t$.

Proposition 5.3. Let $\varepsilon$ be a strict positive real number, $T>0$ a large enough real number, and $u$ be the feedback given in Proposition 5.2.

We assume that $|x(0)| \leq \varepsilon / 2$, where $x=\left(x_{1}, x_{2}, x_{3}\right)^{\prime}$.

Then $(0,0) \in \mathbb{R}^{2} \times \mathbb{R}$ is 2-partially stable in finite-time for the closed loop system (2.2) with $v_{1}$ and $v_{2}$ defined by

$$
v_{1}(x):=\operatorname{sat} \frac{\varepsilon}{2 T}\left\{\left|x_{1}\right|^{\alpha / 2}+\left|x_{2}\right|^{\alpha}\right\}, \alpha \in(0,1) \text { and } v_{2}:=v_{1} u
$$

and therefore $\left|x_{3}(t)\right| \leq \varepsilon, \forall t \geq 0$.

Proof. The proof is similar to the proof of Proposition 3.5.

\subsection{Numerical simulations}

In this subsection we illustrate our design procedure by simulations. It is verified in Figure 5 that the discontinuous stabilizing feedbacks are global two-partially stable in finite-time the Brockett's integrator.

Boundedness of the limit of the state $x_{3}$ for the system. The boundedness of the limit of the state $x_{3}$ is presented in Figure 6. The simulation shows that the limit $c$ satisfies $|c| \leq 6.86$ with the initial condition $x_{0}=(0.8,0.9,0.2)$ and with the saturation $\varepsilon / 2 T=20 / 120(\varepsilon=20, T=60)$.

\subsection{Robust stabilization of perturbed system}

This subsection is devoted to stabilize, by discontinuous finite-time feedback laws, the perturbed control system

$$
\begin{aligned}
& \dot{y}_{1}=u_{1} \cos \theta+\varepsilon u_{1} \sin \theta \\
& \dot{y}_{2}=u_{1} \sin \theta-\varepsilon u_{1} \cos \theta \\
& \dot{\theta}=u_{2} .
\end{aligned}
$$

As shown in Section 4, the system (5.11) is equivalent to

$$
\begin{aligned}
& \dot{z}_{1}=\left(\left(1+\varepsilon^{2}\right) z_{2}+\varepsilon z_{1}\right) v_{1} \\
& \dot{z}_{2}=v_{2} \\
& \dot{z}_{3}=v_{1},
\end{aligned}
$$

the same question of robustness can be asked. 

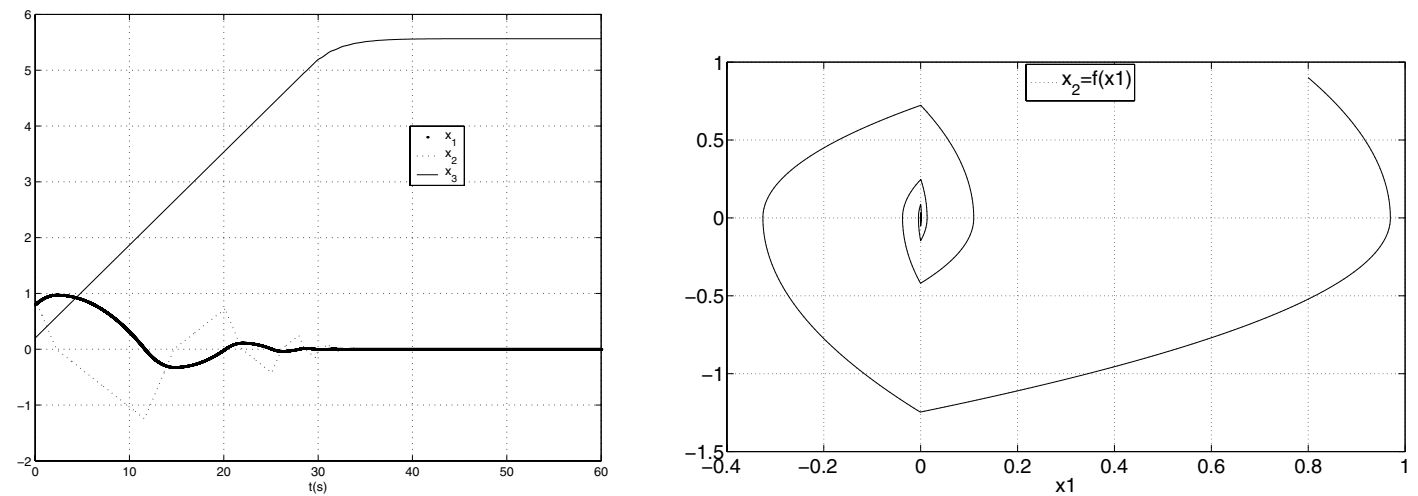

Figure 6 . Trajectories of the state $x_{1}, x_{2}$ and $x_{3}$ (left); motion in the plane $\left(x_{1}, x_{2}\right)$ (right).

Open question. Is the proposed feedback laws (5.1)-(5.2) are robust with respect to the admissible perturbation $\left.g_{\varepsilon}(z)=\left(\left(1+\varepsilon^{2}\right) z_{2}+\varepsilon z_{1}\right), 0,0\right)$ ?

Remark 5.4. Link between local controllability and partial asymptotic stabilization. Throughout the recent years, a great effort has been done to extend the well known relation between controllability and stabilizability for the linear case to the non linear case [16,19,21,23]. Thanks to Kalman theorem [23], controllability for the linear case is equivalent to stabilizability. The situation remains difficult for the nonlinear control systems; it is shown by Brockett that the double integrator (1.2) is controllable however a stabilizing feedback laws does not exist. The same question can be asked for the partial asymptotic stabilizability case: does local controllability or even global controllability implies the partial asymptotic stabilizability? In this remark, we will prove that local controllability does not imply partial asymptotic stabilizability.

Consider the following system

$$
\begin{aligned}
& \dot{x}_{1}=f\left(x_{1}, u_{1}\right) \\
& \dot{x}_{2}=u_{2},
\end{aligned}
$$

where $f(x, u)$ is given as follows [43]

$$
f(x, u)=\left\{\begin{array}{l}
x \text { if }|u| \leq 1 \\
x+\exp \left(\frac{1}{1-u}\right) \text { if } u>1 \\
x-\exp \left(\frac{1}{1+u}\right) \text { if } u<-1 .
\end{array}\right.
$$

It was shown in [43] that (5.14) is locally controllable and a continuous feedback law stabilizing the system does not exist. Clearly, the system (5.13) is locally controllable, since the two subsystems are independent, and (5.13) is not partially asymptotically stabilizable because (5.14) is not Lyapunov stable.

Remark 5.5. We do not know if the combination of Brockett condition and local controllability, even the local controllability in small time STLC, implies the partial asymptotic stabilizability. 


\section{Conclusion}

In this paper, we have studied the finite-time partial stability of a prototype system of nonholonomic control systems which is the benchmark knife edge or the unicycle robot system called also the Brockett's integrator. We have proposed various feedback controllers that achieve the partial asymptotic stabilizability, or the finite-time partial stability of the mobile robot. These feedbacks are Hölderien for the rational partial stability, continuous and homogeneous of negative degree or discontinuous and quasi-homogeneous of negative degree for the finitetime partial stability. From a practical point of view, we have shown that this special stabilization (asymptotic or in finite-time) of the system (1.2) is sufficient. Indeed, for the knife edge, the component $x_{3}$ of the state is the angle that gives the orientation of the vehicle. As we have seen, with the action of our feedback we can place in finite-time the vehicle in the equilibrium position without taking into consideration this angle (this later converges to some position depending on the initial conditions). Clearly, for many cases, this type of partial stabilization is sufficient.

Acknowledgements. We thank Pierre Rouchon for having attracted our attention to the robust stabilizability problem and for useful discussions and comments. We also thank the reviewers for useful comments and suggestions to improve the quality of the paper.

\section{REFERENCES}

[1] D. Aeyels, Stabilization by smooth feedback of the angular velocity of a rigid body. Syst. Control Lett. 5 (1985) 59-63.

[2] D. Aeyels, Stabilization of a class of nonlinear systems by a smooth feedback control. Syst. Control Lett. 5 (1985) $289-294$.

[3] D. Aeyels and M. Szafranski, Comments on the stabilizablity of angular velocity of rigid body. Syst. Control Lett. 10 (1988) $35-39$.

[4] V. Andriano, Global feedback stabilization of the angular velocity of symmetric rigid body. Syst. Control Lett. (1993) 361-364.

[5] A. Astolfi, Asymptotic stabilization of nonholonomic systems with discontinuous control. Ph.D. thesis, Swiss Federal Institute of Thechnology, Zurich (1996).

[6] A. Astolfi, Discontinuous control of nonholonomic systems. Syst. Control Lett. 27 (1996) 37-45.

[7] A. Bacciotti, Local stabilizability of nonlinear control systems. World Scientific (1991).

[8] A. Bacciotti and L. Rosier, Liapunov Functions and Stability in Control Theory. Communications and Control Engineering, Springer-Verlag (2005).

[9] L. Beji, A. Abichou and Y. Bestaoui, Position and attitude control of an underactuated autonomous airship. International Journal of Differential Equations and Applications 8 (2004) 231-255.

[10] M.K. Bennani and P. Rouchon, Robust stabilization of flat and chained systems, in European Control Conf. (1995).

[11] S.P. Bhat and D.S. Bernstein, Finite-time stability of homogenoues systems, in Procceding of the American Control Conference, Albuquerque, New Mexico (1997) 2513-2514.

[12] S.P. Bhat and D.S. Bernstein, Continuous finite-time stabilization of the translational and rotational double integrators. IEEE Trans. Automat. Contr. 43 (1998) 678-682.

[13] S.P. Bhat and D.S. Bernstein, Finite-time stability of continuous autonomous systems. SIAM J. Control Optim. 38 (2000) $751-766$.

[14] S.P. Bhat and D.S. Bernstein, Geometric homogeneity with applications to finite-time stability. Math. Control Signals Syst. 17 (2005) 101-127.

[15] R.W. Brockett, Asymptotic stability and feedback stabilization, in Differential geometric control theory, Progress in Math. 27 (1983) 181-191.

[16] S. Celikovsky and H. Nijmeijer, On the relation between local controllability and stabilizability for a class of nonliner systems. IEEE Trans. Automat. Contr. 42 (1996) 90-94.

[17] F.M. Ceragioli, Discontinuous Ordinary Differential Equations and Stabilization. Tesi di dottorato di ricerca in matematica, Consorzio delle universit'a di Cagliari, Firenze, Modena, Perugia e Siena (1999).

[18] F.M. Ceragioli, Some remarks on stabilization by means of discontinuous feedbacks. Syst. Control Lett. 45 (2002) $271-281$.

[19] J.-M. Coron, A necessary condition for feedback stabilization. Syst. Control Lett. 14 (1990) 227-232.

[20] J.-M. Coron, Global asymptotic stabilization for controllable systems without drift. Math. Control Signals Systems 5 (1992) 295-312.

[21] J.-M. Coron, Relations entre commandabilité et stabilisations non linéaires, in Nonlinear partial differential equations and their applications XI, Collège de France Seminar, Paris (1989-1991), Pitman Res. Notes Math. Ser. 299, Longman Sci. Tech., Harlow (1994) 68-86. 
[22] J.-M. Coron, Stabilization in finite time of locally controllable systems by means of continuous time-varying feedback laws. SIAM J. Control Optim. 33 (1995) 804-833.

[23] J.-M. Coron, Control and Nonlinearity, Mathematical Surveys and Monographs 136. American Mathematical Society (2007).

[24] J.-M. Coron and B. d'Andréa Novel, Smooth stabilizing time-varying control laws for a class of nonlinear systems. Applications to mobile robots, in IFAC Nonlinear Control Systems Design, M. Fliess Ed., Bordeaux, France (1992) 413-418.

[25] J.-M. Coron and E.Y. Keraï, Explicit feedbacks stabilizing the attitude of a rigid spacecraft with two torques. Automatica 32 (1996) 669-677.

[26] J.-M. Coron and J.-B. Pomet, A remark on the design of time-varying stabilizing feedback laws for controllable systems without drift, in IFAC Nonlinear Control Systems Design, M. Fliess Ed., Bordeaux, France (1992) 397-401.

[27] J.-M. Coron and L. Rosier, A relation between continuous time-varying and discontinuous feedback stabilization. J. Math. Systems Estimation and Control 4 (1994) 67-84.

[28] A.L. Fradkov, I.V. Miroshnik and V.O. Nikiforov, Nonlinear and adaptive Control of Complex Systems. Kluwer Academic (2001).

[29] W. Haddad, V. Chellaboina and S. Nersesov, A unification between partial stability of state-dependent impulsive systems and stability theory for time-dependent impulsive systems, in Proc. Amer. Contr. Conf. (2003) 4004-4009.

[30] V. Haimo, Finite time controllers. SIAM J. Control Optim. 24 (1986) 760-770.

[31] H. Hermes, Homogeneous coordinates and continuous asymptotically stabilizing feedback controls, Lecture notes in pure and applied Math. 127, S. Elaydi Ed., Proc. Colorado Springs conf. Marcel Dekker Inc., New York (1990) 249-260.

[32] Y. Hong, Finite-time stabilization and stabilizability of a class of controllable systems. Syst. Control Lett. 46 (2002) $231-236$.

[33] Y. Hong and Z.-P. Jiang, Finite-time stabilization of nonlinear systems with parametric and dynamic uncertainties. IEEE Trans. Automat. Contr. 51 (2006) 1950-1956.

[34] Y. Hong, J. Huang and Y. Xu, On an output feedback finite-time stabilization problem. IEEE Trans. Automat. Contr. 46 (2001) 305-309.

[35] X. Huang, W. Lin and B. Yang, Global finite-time stabilization of a class of uncertain nonlinear systems. Automatica 41 (2005) 881-888.

[36] C. Jammazi, Backstepping and Partial Asymptotic Stabilization. Applications to Partial Attitude Control. International Journal of Control Automation and Systems 6 (2008) 859-872.

[37] C. Jammazi, Finite-time partial stabilizability of chained systems. C. R. Acad. Sci. Paris., Sér. I 346 (2008) 975-980.

[38] C. Jammazi, On the partial attitude control of axisymmetric rigid spacecraft, in Intelligent Systems and Automation: 1st Mediterranean Conference on Intelligent Systems and Automation, AIP Conf. Proc. 1019, H. Arioui, R. Marrouki and H.A. Abbassi Eds., Annaba, Algeria (2008) 302-307.

[39] C. Jammazi, Further results on finite-time partial stability and stabilization. Applications to nonlinear control systems, in Intelligent Systems and Automation: 2nd Mediterranean Conference on Intelligent Systems and Automation, AIP Conf. Proc. 1107, L. Beji, S. Otmane and A. Abichou Eds., Zarzis, Tunisia (2009) 111-116.

[40] C. Jammazi, On a sufficient condition for finite-time partial stability and stabilization: Applications. IMA J. Math. Control Inf. 27 (2010) 29-56.

[41] C. Jammazi and A. Abichou, Partial stabilizability of an underactuated autonomous underwater vehicle, in Proc. in International Conference "System Identification and Control Problems" SICPRO'07, Moscow Institute of Control (2007) 976-986.

[42] H.K. Khalil, Nonlinear Systems. Prentice Hall (2002).

[43] A.L. Kovalev and A.L. Zuyev, On nonasymptotic stabilization of controllable systems, in Proceedings of the 14 International Symposium on Mathematical theory of networks and systems (MTNS), Perpignan, France (2000).

[44] D.A. Lizárraga, P. Morin and C. Samson, Non-robustness of continuous homogeneous stabilizers for affine control systems, in Proceedings of the 38th IEEE Conference on Decision and Control, Phoenix, Arizona, USA (1999) 855-860.

[45] M. Maini, P. Morin, J.-B. Pomet and C. Samson, On the robust stabilization of chained systems by continuous feedback, in Proceedings of the 38th IEEE Conference on Decision and Control, Phoenix, Arizona, USA (1999) 3472-3477.

[46] R.T. M'Closkey and R.M. Murray, Exponential stabilization of driftless nonlinear control systems using homogeneous feedback. IEEE Trans. Automat. Contr. 42 (1997) 614-628.

[47] P. Morin and C. Samson, Time-varying exponential stabilization of a rigid spacecraft with two control torques. IEEE Trans. Automat. Contr. 42 (1997) 528-534.

[48] P. Morin, C. Samson, J.-B. Pomet and Z.-P. Jiang, Time-varying feedback stabilization of the attitude of a rigid spacecraft with two controls. Syst. Control Lett. 25 (1995) 375-385.

[49] E. Moulay, Une contribution à l'étude de la stabilité en temps fini et de la stabilisation. Ph.D. thesis, L'École Centrale de Lille (2005)

[50] P. Morin, J.-B. Pomet and C. Samson, Development of time-varying feedback stabilization of nonlinear systems, in Nonlinear control design symposium NOLCOS (1998) 587-594.

[51] Y. Orlov, Discontinuous systems - Lyapunov Analysis and Robust Synthesis under Uncertainty Conditions. Communications and Control Engineering, Springer-Verlag (2009).

[52] B.E. Paden and S.S. Sastry, A calculus for computing Filippov's differential inclusion with application to the variable structure control of robot manipulators. IEEE Trans. Circuits Systems CAS-34 (1987) 73-82. 
[53] K.Y. Pettersen and O. Egeland, Exponential stabilization of an underactuated surface vessel, in Proc. 35th IEEE Conf. on Decision Control, Kobe, Japan (1996).

[54] Z. Qu, Robust control of nonlinear uncertain systems without generalized matching conditions. IEEE Trans. Automat. Contr. 40 (1995) 1453-1460.

[55] N. Rouche, P. Habets and P. Laloy, Stability Theory by Lyapunov's Direct Method. Applied Mathematical Sciences, SpringerVerlag (1977).

[56] E.P. Ryan, On Brockett's condition for smooth stabilizability and its necessity in a context of nonsmooth feedback. SIAM J. Control Optim. 32 (1994) 1597-1604.

[57] C. Samson, Velocity and torque feedback control of a nonholonomic cart, in Proceedings of International Workshop on Nonlinear and Adaptive Control 162, Springer-Verlag (1991) 125-151.

[58] C. Samson, Control of chained systems: Application to path following and time-varying point-stabilization of mobile robots. IEEE Trans. Automat. Contr. 40 (1995) 64-77.

[59] E.D. Sontag, Mathematical Control Theory: Determinstic Finite Dimensional Systems, Text in Applied Mathematics 6. Springer-Verlag (1998).

[60] E.D. Sontag, Stability and stabilization: Discontinuities and the effect of disturbances, in Nonlinear Analysis, Differential Equations and Control, Proc. NATO Advanced Study Institute, Montreal, F.H. Clarke and R.J. Stern Eds. (1999) 551-598.

[61] E.D. Sontag and H.J. Sussmann, Remarks on continuous feedback, in 19th IEEE Conference on Decision and Control, Albuquerque (1980) 916-921.

[62] W. Su and M. Fu, Robust nonlinear control: beyond backstepping and nonlinear forwarding, in IEEE Conference on decision and control (1999) 831-836.

[63] W. Su and M. Fu, Robust stabilization of nonlinear cascaded systems. Automatica 42 (2006) 645-651.

[64] H.J. Sussmann, Subanalytic sets and feedback control. J. Differential Equations 31 (1979) 31-52.

[65] V.I. Vorotnikov, Partial Stability and Control. Birkhäuser (1998).

[66] V.I. Vorotnikov, Partial stability and control: The state-of-the art and development. Autom. Remote Control 66 (2005) $511-561$.

[67] A.L. Zuyev, On Brockett's condition for smooth stabilization with respect to part of variables, in Proc. European Control Conference ECC'99, Karlsruhe, Germany (1999).

[68] A.L. Zuyev, On partial stabilization of nonlinear autonomous systems: Sufficient conditions and examples, in Proc. of the European Control Conference ECC'01, Porto, Portugal (2001) 1918-1922. 